\title{
Juristinnen? Nein, danke! -
}

\author{
Die Juristenausbildung als Mittel der Ausgrenzung von Frauen aus rechtshistorischer Perspektive
}

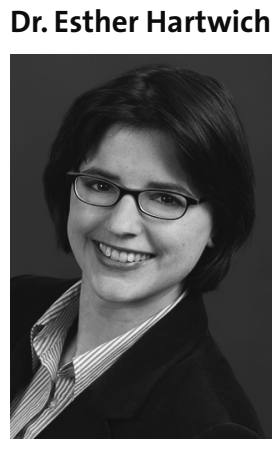

Mitglied im Vorstand des Landesverbandes Berlin, Referatsleiterin Bildungsrecht beim Deutschen Industrieund Handelskammertag, Berlin

\section{Dr. Inbal Steinitz}

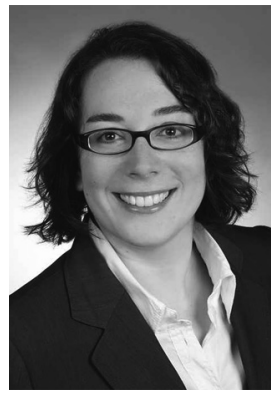

Kollegiatin des Internationalen Max Planck Forschungskollegs für vergleichende Rechtsgeschichte, Rechtsassessorin, Berlin
Die Juristenausbildung diente seit ihrer Professionalisierung immer wieder als Ausgrenzungsmittel gegenüber bestimmten Personengruppen. In der DDR beispielsweise garantierten sowohl das Auswahlverfahren als auch der Inhalt der Ausbildung, dass nur parteilinientreue Bewerber zu einem Studium zugelassen wurden und eine juristische Ausbildung durchlaufen konnten. ${ }^{1}$ Die Gruppe, die im Laufe der Geschichte am stärksten ausgegrenzt wurde, war die der Frauen. Hatten sich Frauen am Anfang des 20. Jahrhunderts mühsam und schrittweise ihren Zugang zu Studium und juristischem Vorbereitungsdienst erkämpft, so wurden diese Errungenschaften in der Zeit des Nationalsozialismus wieder rückgängig gemacht und der Zugang zum Referendariat erschwert. Erst nach dem zweiten Weltkrieg wurde sowohl in der Bundesrepublik Deutschland als auch in der DDR die juristische Ausbildung wieder vollständig für Frauen geöffnet. In Zeiten des Bologna-Prozesses und der aktuellen Reformdiskussionen ${ }^{2}$ lohnt ein Blick auf die historische Entwicklung der Juristenausbildung mit Fokus auf die verschiedenen Ausgrenzungsmechanismen, von denen Frauen in besonderem Maß betroffen waren.

\section{Die historische Entwicklung der juristischen Ausbildung}

Die Geschichte der juristischen Ausbildung beginnt im 11. Jahrhundert. Die Römer kannten zwar den Beruf des Juristen, dieser ging jedoch zusammen mit dem Römischen Reich unter. ${ }^{3}$ In der Folge lag die Rechtspflege in den Händen von Personen ohne fachspezifische Schulung; des Schreibens fähige Geistliche übernahmen Verwaltungsaufgaben und die Rechtsprechung erledigten so genannte rechtskundige Männer. Erst die Unterrichtung des wiederentdeckten corpus iuris civilis an der Universität von Bologna in der Wende vom 11. zum 12. Jahrhundert markiert den Beginn einer juristischen Ausbildung. Zunächst kamen die Studenten aus ganz Europa zum Rechtsstudium nach Bologna. Seit dem 14. Jahrhundert wurden auch in Deutschland Universitäten gegründet, die ein rechtliches Studium anboten. Ziel des Studiums war die Heranbildung wissenschaftlicher Rechtsgelehrter, das für die Praxis relevante Territorialrecht wurde hingegen nicht unterrichtet. Um die Rechtsgelehrten auch für die Praxis zu qualifizieren, begann Preußen ab dem 18. Jahrhundert schrittweise einen ver- bindlichen Vorbereitungsdienst mit staatlichen Eingangs- und Abschlussprüfungen zu entwickeln. Erst beschränkt auf Richter wurde er im 19. Jahrhundert nach und nach auf Anwälte, Notare, Staatsanwälte und schließlich auch auf Verwaltungsjuristen ausgeweitet. Ende des 19. Jahrhunderts erhielt die preußische Ausbildung - die 1877 Grundlage der gesamtdeutschen wurde - die bis heute geltende Struktur mit einem an das Studium anschließenden ersten Staatsexamen und dem mit dem zweiten Staatsexamen abschließenden Referendariat.

Die staatliche Regelung eröffnete die Möglichkeit, den Zugang zur juristischen Ausbildung zu kontrollieren und diese als Mittel zur Ausgrenzung bestimmter Gruppen zu nutzen. Waren Frauen im 19. Jahrhundert bereits vom Studium ausgeschlossen, wurde anderen Gruppierungen erst der $\mathrm{Zu}$ gang zum staatlichen Vorbereitungsdienst verwehrt. So bestand beispielsweise für Juden zwar die Möglichkeit eines rechtswissenschaftlichen Studiums, zum Referendariat wurden sie in Preußen indes erst nach der Märzrevolution 1848 zugelassen. ${ }^{4}$ Die vierjährige unbesoldete Referendariatszeit bewirkte zudem eine soziale Auslese, die den Juristenstand für viele Jahrzehnte prägen sollte. Zulassungsvoraussetzung war ein so genanntes Sustentationsattest, mit dem nachgewiesen werden musste, dass sich der angehende Jurist in der langen Ausbildungszeit selbst ernähren konnte und sein Unterhalt gesichert war. Nicht alle Jurastudenten konnten es sich leisten, vier Jahre lang ohne Gehalt zu leben. Die „gute Herkunft“ wurde dementsprechend zu einer Voraussetzung der Ausbildung zum Volljuristen. Erst in der Weimarer Republik wurde

1 Christian Grüneberg, Juristenausbildung in der DDR, DRiZ 1990, S. 295 f.; Siehe dazu ausführlich Malgorzata Liwinska, Juristische Ausbildung in der DDR im Spannungsfeld von Parteilichkeit und Fachlichkeit, Berlin 1997.

2 Barbara Dittmann, Plädoyer für Bologna? Justizminister im Kreuzverhör, djbZ 2/2008, S. $101 \mathrm{ff}$.

3 Zur historischen Entwicklung der Juristenausbildung einführend Hans Hattenhauer, Juristenausbildung Geschichte und Probleme, JuS 1989, S. 513 ff. und Nicolas Lührig, die Diskussion über die Reform der Juristenausbildung von 1945 bis 1995, Frankfurt am Main 1997, S. 26 ff. mit weiterführenden Literaturhinweisen.

4 Ina Ebert, Die Normierung der juristischen Staatsexamina und des juristischen Vorbereitungsdienstes in Preußen (1849-1934), Berlin 1995, S. 344 ff. Die Übertragung eines Richteramtes wurde den Juden kraft Gesetz bis 1866 verweigert. Auch nach der rechtlichen Gleichstellung wurden sie in ihrer Berufsausübung weiter faktisch diskriminiert. 
1919 die Voraussetzung des Sustentationsattestes zurückgenommen und den Referendaren auch die Ausübung von bezahlten Nebentätigkeiten gestattet.

\section{Die Zulassung von Frauen zur juristischen Ausbildung}

Die Frauen konnten sich die Zulassung zum juristischen Vorbereitungsdienst erst in der Weimarer Republik erkämpfen, zunächst galt es den Zugang zum Studium zu erstreiten. Diese Forderung erhoben sie erstmals öffentlich während der Märzrevolution 1848. ${ }^{5}$ Im 19. Jahrhundert strebten Frauen der Mittel- und Oberschicht zunehmend nach höherer Bildung, um ihrem neuen weiblichen Selbstverständnis Ausdruck zu verleihen und eine berufliche Tätigkeit ausüben zu können. 1867 erklärte der Allgemeine Deutsche Frauenverein die Zulassung zum Studium zum vorrangigen Ziel der bürgerlichen Frauenbewegung. Gegen diese Forderung wehrten sich die Universitäten nicht nur wegen der Vorurteile gegenüber den intellektuellen Fähigkeiten der Frauen, sondern auch, um die Entwicklung eines universitären Großbetriebes zu verhindern. ${ }^{6}$ Gegen Ende des 19. Jahrhunderts strömten vermehrt Studenten aus dem bürgerlichen Milieu an die Universitäten. Durch die Zulassung von Frauen befürchteten die Fakultäten diese Entwicklung zu begünstigen. Der Aufnahme eines Studiums durch Frauen stand außerdem entgegen, dass sie den für Männer üblichen Weg zur Erlangung des Abiturs nicht beschreiten durften. Erst am Ende des 19. Jahrhunderts wurde den ersten Mädchen erlaubt, als Externe an den Abitursprüfungen teilzunehmen. 1896 legten die ersten sechs Frauen in Deutschland die allgemeine Hochschulreife $a b$, nachdem sie private Gymnasialkurse besucht hatten. ${ }^{7}$

Im Jahr 1900 ermöglichte Baden als erstes Land Frauen, an den Universitäten zu studieren. ${ }^{8}$ Die Zulassung zum Studium bedeutete allerdings noch nicht die Zulassung zu den Staatsexamina und zum Referendariat. Der einzige den Frauen mögliche Studienabschluss war die Promotion. Weil ihnen mit einem solchen Abschluss die Ausübung eines klassischen juristischen Berufes verwehrt war, war der Anteil der Frauen an rechtswissenschaftlichen Fakultäten zunächst noch sehr gering. So studierten etwa 1908 in ganz Deutschland lediglich acht Frauen Jura. ${ }^{9}$ Ziel der Frauenverbände war es daher nun, die Aufnahme der Frauen ins Referendariat zu erreichen, um ihnen das Erlangen eines vollwertigen Abschlusses zu ermöglichen. Im Jahr 1912 ließ erstmals Bayern Frauen zum ersten Staatsexamen zu. Das Land verweigerte ihnen jedoch die Aufnahme in den Vorbereitungsdienst mit der Begründung, dass die Frauen nach dem geltenden Reichsrecht sowieso nicht den Beruf einer Richterin, Staatsanwältin oder Anwältin ausüben dürften. ${ }^{10}$ So argumentierten auch die anderen Länder, auf Reichsebene wiederum berief man sich auf die Ausbildungshoheit der Länder.

Die rechtliche Ungleichbehandlung änderte sich mit der Weimarer Republik. Die Weimarer Verfassung gewährte den Frauen das aktive und passive Wahlrecht (Art. 22 WRV), die grundsätzliche rechtliche Gleichstellung mit den Männern (Art. 109 II WRV) sowie die Beseitigung der Benachteiligung im öffentlichen Dienst (Art. 128 II WRV). Trotz der verfassungsrechtlichen Gleichstellung war die Anpassung der Reichsgesetze nicht selbstverständlich. ${ }^{11}$ Zum einen bestanden erhebliche Vorurteile gegenüber der Fähigkeit der Frauen, unparteiisch als Richterinnen urteilen zu können. Auf dem Richtertag im Jahr 1921 führte ein Landgerichtsdirektor dazu etwa Folgendes aus: „Die aus der seelischen Eigenart der Frau sich ergebenden Gefahren erhalten eine erhebliche Steigerung in der Zeit der Monatsperiode, der Schwangerschaft und der Wechseljahre. In dieser Zeit befindet sich nämlich die Frau [...] in einem erhöhten Zustand der Reizbarkeit" . ${ }^{12}$ Stärker noch als diese Vorurteile wogen die Ängste vor einer durch Frauen zunehmenden Konkurrenz. So wurde während der Gesetzgebungsverfahren immer wieder auf das bereits bestehende Überangebot an Juristen hingewiesen. Durch intensive Bemühungen der weiblichen Abgeordneten und der Frauenverbände - darunter auch der 1914 gegründete Deutsche Juristinnenverein - gelang es trotzdem, im Juli 1922 mit dem „Gesetz über die Zulassung der Frauen zu den Ämtern und Berufen der Rechtspflege“ Frauen das Tor zu allen juristischen Berufen zu öffnen. Infolgedessen mussten nun auch die Länder ihre Ausbildungsordnungen ändern. Aufgrund der neuen beruflichen Perspektiven entschieden sich immer mehr Frauen für ein Jurastudium. 1923 betrug ihr Anteil an den in Deutschland Rechtswissenschaft Studierenden mit 580 Studentinnen 6, 1 Prozent. Wegen der anhaltenden faktischen Diskriminierung im juristischen Berufsleben nahm ihre Zahl jedoch bald wieder ab. Schon 1926 sank ihr Anteil mit 378 Jurastudentinnen auf nur noch 2,5 Prozent. ${ }^{13}$

\section{Die Ausgrenzung der Frauen von der Juristenausbildung im Nationalsozialismus}

Nach der Öffnung der Juristenausbildung für die Frauen in der Weimarer Republik, wurde ihnen die Absolvierung der ju-

5 Kristine von Soden/Gaby Zipfel (Hrsg.), 70 Jahre Frauenstudium, Frauen in der Wissenschaft, Köln 1979.

6 Kristine von Soden, Auf dem Weg in die Tempel der Wissenschaft. Zur Durchsetzung des Frauenstudiums im Wilhelminischen Deutschland, in: Ute Gerhard (Hrsg.), Frauen in der Geschichte des Rechts: von der frühen Neuzeit bis zur Gegenwart, München 1997, S. $621 \mathrm{f}$.

7 Deutscher Juristinnenbund (Hrsg.), Juristinnen in Deutschland. Die Zeit von 1900 bis 2003, 4. Auflage, Baden-Baden 2003, S. 13; Ursula Rust, Zur Situation von Frauen in der juristischen Ausbildung und an den juristischen Fakultäten, in: Ursula Rust (Hrsg.), Juristinnen an den Hochschulen - Frauenrecht in Lehre und Forschung, BadenBaden 1997, S. 92.

8 Es folgten Bayern 1903, Württemberg 1904, Sachsen 1906, Thüringen 1907, Hessen 1908, Preußen 1908 und Mecklenburg 1909.

9 Die Zahlenangaben stammen aus Stefan Bajohr / Kathrin RödigerBajohr, Die Diskriminierung der Juristin in Deutschland bis 1945, KJ 1980, S. 39 ff. sowie Ursula Rust, a.a.O., S. 92 ff.

10 Inge Beate Schwanecke, Die Gleichberechtigung der Frau unter der Weimarer Reichsverfassung, Heidelberg 1977, S. 22 ff.

11 Zum Verlauf des Gesetzgebungsverfahren vgl. Gisela Wild, Frauen in der Rechtspflege, in: Jan Albers (Hrsg.), Recht und Juristen in Hamburg, Köln 1994, S. 267 ff. und Juristinnen in Deutschland, a.a.O., S. $17 \mathrm{ff}$.

12 DRiZ 1921, S. 200.

13 Zahlen aus Stefan Bajohr / Kathrin Rödiger-Bajohr, a.a.O., S. 39 ff. und Ursula Rust, a.a.O., S. 92 ff. 
ristischen Ausbildung durch die nationalsozialistischen Machthaber wiederum erschwert. So wurde ihnen beispielsweise der Besuch der Referendarslager, der seit 1933 fester Bestandteil des juristischen Vorbereitungsdienstes war, untersagt. ${ }^{14}$ Auch die Neufassung der Justizausbildungsordnung von 1934 konnte so ausgelegt werden, dass nur Männer zu den Staatsexamina zugelassen werden konnten. ${ }^{15}$ Den Frauen wurde der Zugang zu den Prüfungen dennoch nicht verweigert, da die Beschränkungen in der Berufsausübung so erheblich waren, ${ }^{16}$ dass sich die Anzahl der Jurastudentinnen ohnehin erheblich reduzierte. Die berufliche Ausgrenzung der Juristinnen im Nationalsozialismus hatte zur Folge, dass nach 1945 Bewerberinnen für den Justizdienst oft wegen fehlender Berufserfahrung abgewiesen wurden, hatten sie doch im Gegensatz zu ihren männlichen Kollegen keine lückenlose juristischen Werdegänge vorzuweisen. ${ }^{17}$ Zumindest in rechtlicher Hinsicht wurden die Zulassungsbeschränkung für Juristinnen aufgrund ihres Geschlechts in Ausbildung und Beruf nach Kriegsende beseitigt. Gerade im Zug der aktuellen Debatten über möglichen Reformen der Juristenausbildung sollte indes nicht außer Acht gelassen werden, dass die Ausbildungsregeln nach wie vor Ausgrenzungspotential besitzen.

14 Folker Schmerbach, Das „Gemeinschaftslager Hanns Kerrl“ für Referendare in Jüterbog 1933-1939, Berlin 2008, S. 40.

15 Stefan Bajohr / Kathrin Rödiger-Bajohr, aaO., S. 46

16 Vgl. hierzu Juristinnen in Deutschland, a.a.O., S. 26 ff. und Ursula Rust, a.a.O., S. $96 \mathrm{f}$.

17 Folker Schmerbach, a.a.O., S. 35 .

\section{Frauenrechte in der Türkei - ein geschichtlicher Überblick -}

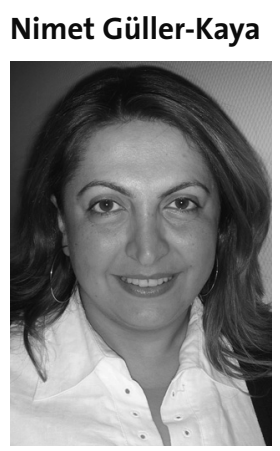

Wissenschaftliche Mitarbeiterin; Teamleiterin und Projektmanagerin, Köln/ Istanbul
Auf dem Gebiet der heutigen Türkei lebten viele verschiedene Völker - von den Hethitern Römern und Griechen bis zu den Seldschuken, Turkmenen und vielen anderen. ${ }^{1}$ Diese Völker hatten sehr unterschiedliche Frauenbilder. Hier geht es um die Geschichte der türkischen Frauen nach der Islamisierung und nach der Entstehung des osmanischen Reiches im Jahre 1299. ${ }^{2}$

Grundlage des osmanischen Rechtssystems war das islamische Gesetz. ${ }^{3}$ Bestand eine Gesetzeslücke, wurden ergänzend weltliche Gesetze durch den Sultan erlassen. Der Koran regelte sowohl das gesellschaftliche Zusammenleben als auch das Rechtssystem. Die Rollen der Geschlechter sowie die soziale und rechtliche Stellung der Frauen waren genauestens festgelegt. Der Koran gab somit vor 14 Jahrhunderten den Frauen Rechte, an die damals sonst nicht zu denken war, weder in der arabischen Welt noch in Europa, wo Frauen noch als Wesen ohne Seele bezeichnet, kein Erb-, Scheidungs- und Unterhaltsrecht hatten oder aber auch als Hexen verbrannt wurden.

Der Islam gab den Frauen das Recht auf Eigentum und Erbe. Jede Frau hatte das Recht auf einen Anteil des Erbes, jedoch nur auf die Hälfte dessen, was die Söhne bekamen. Zudem konnte die Frau ihr Eigentum auch in der Ehe behalten und ihr Geld für ihre eigenen Wünsche ausgeben, da nur die Männer verpflichtet waren, den Lebensunterhalt der Familie zu sichern. Auch wurden ein Mindestalter für die Eheschließung, das Scheidungs- und Unterhaltsrecht eingeführt. Es war sogar Vorschrift, dass der islamische
Mann sich nur dann bis zu vier Frauen nehmen durfte, wenn er sie alle ernähren und gleich lieben konnte, eine positive Entwicklung in der damaligen arabischen Welt, in der Männer sich selten mit nur vier Frauen begnügten und sich jederzeit ohne jegliche Verpflichtungen von den Frauen trennen konnten. Jetzt bedeutete jede Heirat auch die Verpflichtung, für die Frau und die Kinder zu sorgen.

Jedoch entsprach die Wirklichkeit im osmanischen Reich nicht ganz dem islamischen Recht. Zwar richteten sich in dieser theokratischen osmanischen Ordnung die Rechte der Frauen nach dem Koran, aber die meisten Frauen waren zahlreichen

1 Zur Geschichte der Türkei siehe: Dimitri Kitsikis: L'Empire ottoman; 3. Auflage, Paris 1994; Udo Steinbach: Geschichte der Türkei, München 2000.

2 Mit der Gründung des seldschukischen Reiches im Jahre 1071 begann das Eindringen der Turkmenen in die heutige Türkei. Das Seldschukenreich in Kleinasien wurde sehr bald in viele kleine Fürstentümer und Stämme zerschlagen. Der Herrscher eines dieser „Beyliks" turkmenischer Herkunft und islamischen Glaubens war Osman I. Um 1299 erklärte Osman I die Unabhängigkeit seines Beyliks vom Reich der Seldschuken. Dieses Jahr wird traditionell auch als das Gründungsjahr des Osmanischen Reiches - benannt nach Osman I- anerkannt. Nach und nach gewannen Osman und seine Nachfolger die Herrschaft über die benachbarten Gebiete und mit der Eroberung Konstantinopels im Jahre 1453 nahm die Gründung des osmanischen Reiches seinen bekannten Lauf.

3 Die islamischen Glaubenssätze, die der Prophet Mohammed im Auftrag Gottes empfing, stammen aus einer Zeit um 622 n. Chr. und wurden nach etwa zwanzig Jahren nach dem Tode des Propheten im Koran niedergeschrieben. Siehe auch Küng, Hans: Der Islam: Geschichte, Gegenwart, Zukunft; München 2004; Elger, Ralf: Islam; Frankfurt 2002 